\title{
Diagnostische Handgelenksarthroskopie und minimalinvasive therapeutische Möglichkeiten
}

\author{
Oliver Lotter, Hans-Eberhard Schaller
}

\section{Zusammenfassung}

Die Arthroskopie erlaubt nach abgeschlossener Bildgebung (Röntgen, CT und/oder MRT) bei strenger Indikationsstellung eine risikoarme und schonende Methode zur Abklärung von Krankheitszuständen am Handgelenk. Die technischen Voraussetzungen zur Durchführung einer Handgelenksspiegelung sind aufwendig, dies wird jedoch durch kompatible Systeme für Arthroskopien an anderen Gelenken (Knie, Schulter, Ellenbogen usw.) relativiert. Die im Rahmen einer diagnostischen Handgelenksarthroskopie erhobenen Befunde führen nicht selten zur Indikationsstellung für weitere therapeutische Maßnahmen. Diese stellten früher die Domäne des offenen Vorgehens mit Eröffnen des Gelenks (Arthrotomie) dar. Heutzutage werden immer mehr solcher Eingriffe arthroskopisch und einzeitig zusammen mit dem vorangestellten diagnostischen Teil der Operation vorgenommen.

\section{Einleitung}

Die Arthroskopie (Synonym: Gelenkspiegelung, von griechisch arthros $=$ Gelenk und skopein = schauen) stellt eine minimalinvasive chirurgische Technik dar, bei der eine Sonde (Arthroskop) durch einen kleinen Zugang in Gelenke eingebracht wird. Die Betrachtung des Operationsgebiets erfolgt über einen Monitor. In den 1970er-Jahren wurde von Karl Storz das erste leistungsfähige Arthroskop hergestellt. Es war eine Kombination des Stablinsensystems von H.H. Hopkins mit einer Lichtübertra-

OP-JOURNAL 2015; 31: 108-112

(c) Georg Thieme Verlag KG Stuttgart · New York DOI http://dx.doi.org/10.1055/s-0035-1545973

After preoperative diagnostic clarification (radiographs, CT-scan, MRI), wrist arthroscopy represents a risk-less and gentle method for clarification of pathological conditions of the wrist. There are elaborate technical requirements for carrying out wrist arthroscopy. Costs can be mainly reduced by using compatible systems for arthroscopies of other joints such as the knee, the shoulder, the elbow and others. Often, the arthroscopic findings will decide the further proceeding and not seldom therapeutic intervention is indicated. In the beginning of wrist arthroscopy, these were the hallmark of open joint surgery. Today, however, an increasing number of therapeutic interventions are undertaken arthroscopically in one session together with the preceding diagnostic part of the surgery.

\section{Diagnostic Wrist Arthroscopy and Minimally Invasive Therapeutic Options}

\section{Vorbereitung der Handgelenksarthroskopie}

Vor Beginn der Operation, welche üblicherweise in regionaler Plexusanästhesie ambulant durchgeführt werden kann, wird der Patient in Rückenlage auf dem Operationstisch gelagert. Der Arm kann zudem vorübergehend auf einem Handtisch positioniert werden, was das Anlegen der Blutsperre, die Haarentfernung mittels Clipper und das sterile Ablegen nach dem Abwaschen erleichtert. Die Abdeckung erfolgt praktischerweise mit einem großen Lochtuch, welches nur den Arm freilässt. Hier empfiehlt es sich, den Arm bis möglichst über den Ellenbogen nach proximal zu desinfizieren, um schließlich das Lochtuch bis knapp an die Oberarmblutsperre zu bringen. Nach Auswickeln des Armes mit einer gängigen Esmarch-Binde wird die Manschette bei Blutleere auf 75-100 mmHg höher als der systolische Wert des Patienten aufgeblasen, üblicherweise bei $250 \mathrm{mmHg}$ [7].

Zeige- und Mittelfinger der betroffenen Hand werden nun mit Mädchenfängern überzogen (wir bevorzugen Nylon gegenüber Drahtmodellen, welche eine schlechtere Druckverteilung an den Fingern bewirken) oder alternativ in einer Handplatte fixiert (Abb.1). Nun wird das zuvor an den Operationstisch montierte Traktionssystem (Arthroskopieaufhängung) komplettiert, indem je nach Modell ein Stempel auf den Oberarm aufgebracht und Gewichte zum Zug am Handgelenk (rund $5 \mathrm{~kg}$ ) angebracht werden, nachdem die Handfixierung in das System eingespannt worden ist.

Die erste Arthroskopie des Handgelenks wurde im Jahr 1979 durchgeführt, erlangte jedoch erst Mitte der 1980er-Jahre breite Anwendung als diagnostisches Verfahren.

Bis zum heutigen Tag hat sich die Handgelenksarthroskopie als zunächst rein diagnostisches Tool zu einer Möglichkeit mit zunehmender Anzahl an therapeutischen Indikationen weiterentwickelt [3].
Die übrigen vorbereitenden Maßnahmen, insbesondere die Bedienung der Konsole am Arthroskopieturm, der Kamerabezug, die Fixierung der Schläuche an der Abdeckung bzw. am Instrumententisch und die Einstellung der Kamera (Schärfe, Weißabgleich usw.) unterscheiden sich nur unwesentlich vom Ablauf bei Gelenkspiegelungen anderer Gelen- 
ke. Operateur und ggf. Assistent positionieren sich auf der kopfwärtigen Patientenseite des Armtischs, die instrumentierende Person stellt oder setzt sich neben den Operateur, während der Instrumententisch in Verlängerung des Armtischs steht. Somit haben alle teilhabenden Personen während der Spiegelung Sicht auf den Monitor.

Die Minimalausstattung eines diagnostischen Arthroskopiesiebs beinhaltet das 1,9-mm- oder 2,4-mm-Arthroskop mit Trokar, das zugehörige Lichtkabel, einen Tasthaken, ein 11er- oder 15er-Skalpell, ein gebogenes stumpfes Moskitoklemmchen, eine feine Präparierschere sowie Nadelhalter und Pinzette.

Des Weiteren sollte Zugriff auf ein Shaver-Handstück mit Shaver- und Knochenfräsaufsatz, Fasszangen und Punches mit verschiedenen Schnittrichtungen sowie ein Handstück für elektrothermales Schrumpfen/Veröden vorhanden sein (Abb. 2 und 3).

Die Handgelenksspiegelung kann trocken oder feucht durchgeführt werden. Wir bevorzugen bei guter Übersicht Erstere mit der Option des Wechsels auf eine feuchte Arthroskopie bei schlechter Einsicht ins Gelenk, z.B. aufgrund eines Gelenkergusses, störender Synoviazotten oder eines engen Gelenkspalts zur weiteren Aufdehnung. Des Weiteren sollten therapeutische Maßnahmen, welche mit Hitzeentwicklung einhergehen (s.u.), unter den Bedingungen einer laufenden Gelenkspülung durchgeführt werden. Ringer-Laktat bietet als physiologische Flüssigkeit dabei die besten Voraussetzungen für eine feuchte Arthroskopie. Die Flüssigkeitszufuhr erfolgt über den Arthroskopschaft, an welchen ein Infusionssystem mit Heidelber-

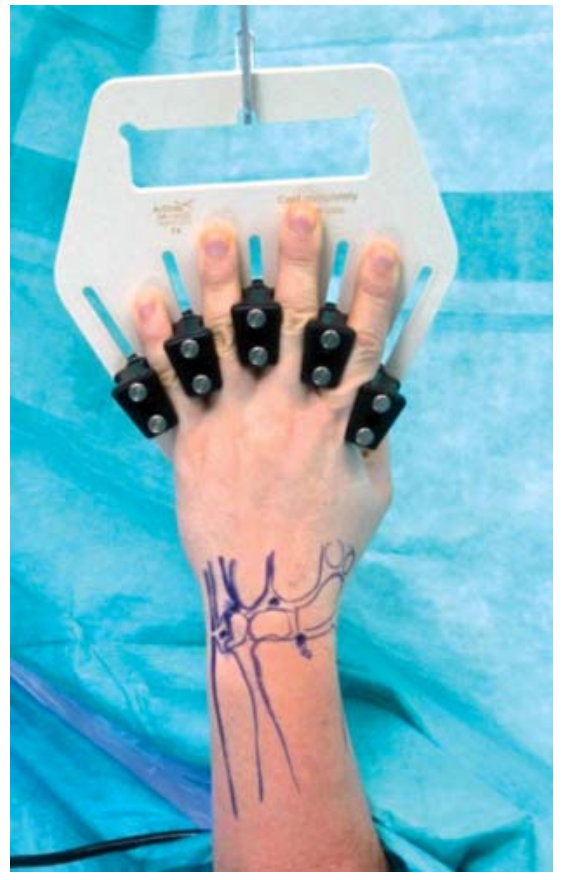

Abb. 1 Aufhängung der Hand in einer Handplatte.

ger Verlängerung oder ein Rollenpumpensystem angeschlossen wird (Abb. 3). Für den kontinuierlichen Abfluss applizieren wir eine gelbe Einmalinjektionskanüle der Größe $1(0,9 \times 40 \mathrm{~mm})$, um insbesondere beim Wechsel von trocken auf feucht häufig störende Luftblasenbildung zu minimieren.

\section{Durchführung der Handgelenksarthroskopie}

Die Handgelenksarthroskopie besteht in ihrer kompletten Durchführung aus 2 Teilbereichen: 1. die eigentliche Handgelenksarthroskopie (radiokarpal und ulnokarpal) und 2. die mediokarpale Arthroskopie zwischen proximaler und distaler Handwurzelreihe.
Zunächst werden sog. Portale in Form von weichen Stellen („soft spots“), meistens am dorsalseitigen Handgelenk, aufgesucht. Hierbei orientiert sich der Operateur an Landmarken, welche das Auffinden erleichtern. Diese Zugangsstellen liegen so, dass Sehnen-, Nerven- und Knochenläsionen auf ein Mindestmaß reduziert werden. Gängige Portale befinden sich z.B. zwischen dem 3. und 4. Strecksehnenfach sowie radial oder ulnar des 6. Strecksehnenfachs (Abb. 4 und 5).

Nach Markierung dieser Zugänge und Punktieren des Gelenks an diesen Stellen mit einer Einmalinjektionskanüle der Größe $1(0,9 \times 40 \mathrm{~mm})$ erfolgt eine longitudinale, etwa $3 \mathrm{~mm}$ lange Inzision der Haut sowie das ebenfalls längsgerichtete Spreizen mit der Präparierschere. Die Schere wird nun um $90^{\circ}$ gedreht, um mit der Biegung nach unten vorsichtig die Handgelenkskapsel zu perforieren. Im Weiteren erfolgt der Wechsel auf ein Moskitoklemmchen, mit dem die Handgelenkskapsel so aufgedehnt wird, dass der Schaft mit Trokar ohne großen Widerstand eingeführt werden kann. Nach Entfernen des Trokars wird das Arthroskop über den Schaft ins Gelenk vorgeschoben (Abb.6).

\section{Radiokarpale und ulnokarpale Handgelenksarthroskopie}

Nach Fokussieren der Kamera und Einstellen des Sichtfelds beginnt nun ein standardisiertes Schema zur Beurteilung des Gelenksitus und dessen Pathologien. Üblicherweise wird die Arthroskopie im Handgelenk radiokarpal begonnen, indem der Griffelfortsatz der Speiche (Processus styloideus radii) betrachtet wird (Abb. 7). Das Interesse gilt hierbei, ebenso wie bei der folgenden Betrachtung der

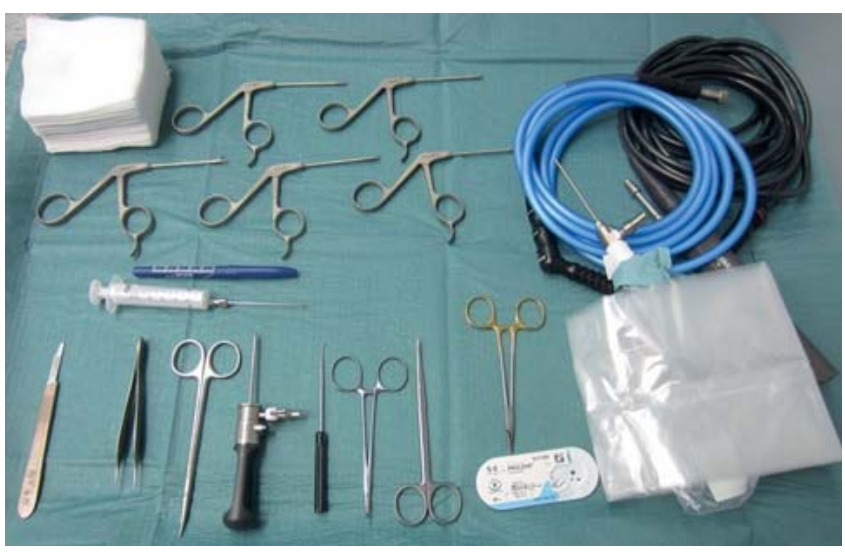

Abb. 2 Instrumententisch.

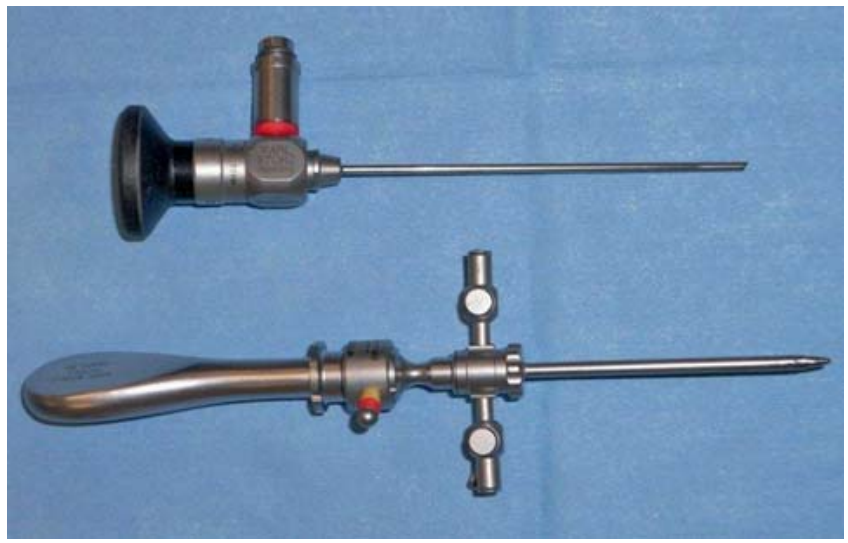

Abb. 3 Arthroskop mit Trokar und Zufuhr für Ringer-Laktat bei feuchter Arthroskopie. 


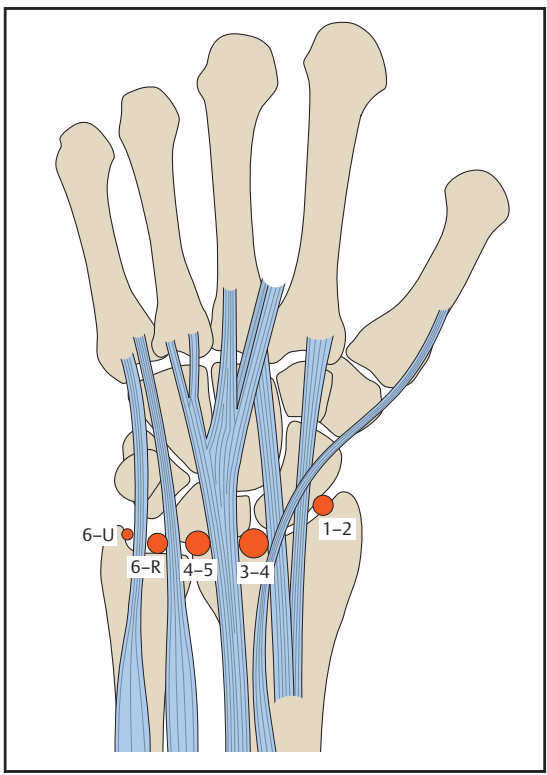

Abb. 4 Radiokarpale und ulnokarpale Portale [1].

Radiusgelenkfläche mit korrespondierendem Kahnbein und Mondbein (proximale Handwurzelreihe), insbesondere dem Knorpelüberzug und dessen Beschaffenheit. Des Weiteren werden die Auskleidung der Gelenkinnenhaut mit möglichen Entzündungszeichen (Synovialitis) (Abb. 8), die palmaren Handgelenksbänder (Abb. 9) sowie das wichtige skapholunäre Band zwischen Kahn- und

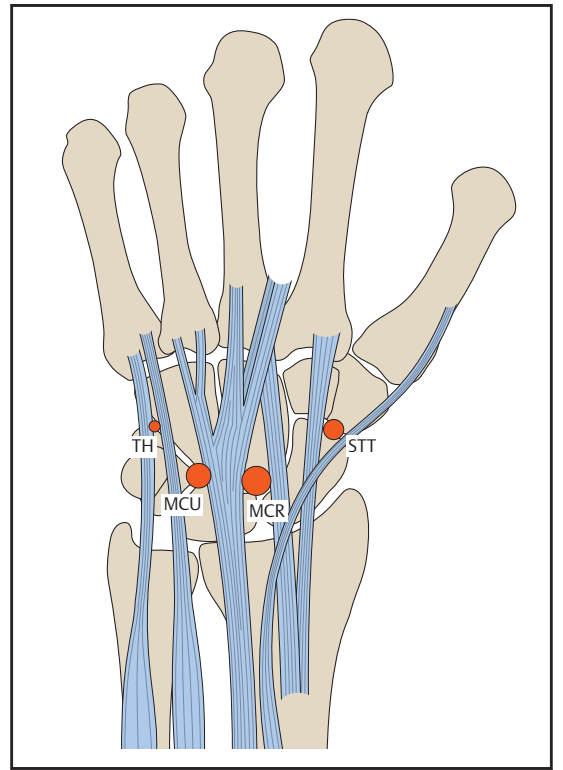

Abb. 5 Mediokarpale Portale [1].

Mondbein beurteilt. Nach Einschwenken in das ulnokarpale Kompartiment des Handgelenks gilt das Interesse v.a. dem triangulären fibrokartilaginären Komplex $($ TFCC $=$ ulnokarpaler Komplex $=$ Discus triangularis), welcher sich in normalem Zustand als Meniskushomolog zwischen dem ulnaren Rand der Speiche und dem Ellengriffelfortsatz (Processus styloideus ulnae) dreiecksförmig wie ein
Segel ausspannt (Abb.10). Er gilt als wichtigster Stabilisator des körperfernen Gelenks zwischen Elle und Speiche und verursacht bei Läsionen insbesondere Schmerzen bei Umwendbewegungen im Handgelenk. Spätestens an diesem Punkt der Operation benötigt der Operateur die Hilfe des Assistenten bzw. der/des Instrumentierenden, da für die Beurteilung des TFCC ein weiterer Zugang für einen Tasthaken vonnöten ist. Hierzu übergibt der Operateur vorübergehend die Kamera, um das 2. Portal wie oben beschrieben anzubringen. Indem schließlich mit dem Tasthaken der TFCC nach unten gedrückt wird, bekommt der Operateur eine Information darüber, ob das „TFCC-Segel“ ausreichend gespannt, oder ob es durch eine Pathologie zum Verlust dieses sog. „Trampolineffekts“ gekommen ist.

\section{Mediokarpale Handgelenksarthroskopie}

Bei der mediokarpalen Handgelenksarthroskopie wird die Region zwischen den Gelenkflächen der proximalen und distalen Handwurzelreihe betrachtet, um dort lokalisierte Veränderungen beurteilen zu können. So ist z.B. die Beurteilung des wichtigen Bandes zwischen Kahnbein und Mondbein (scapholunäres Band) nur durch eine kombinierte radiokarpale/ulnokarpale und mediokarpale Gelenkspiegelung möglich (Abb.11). Das Kamera-
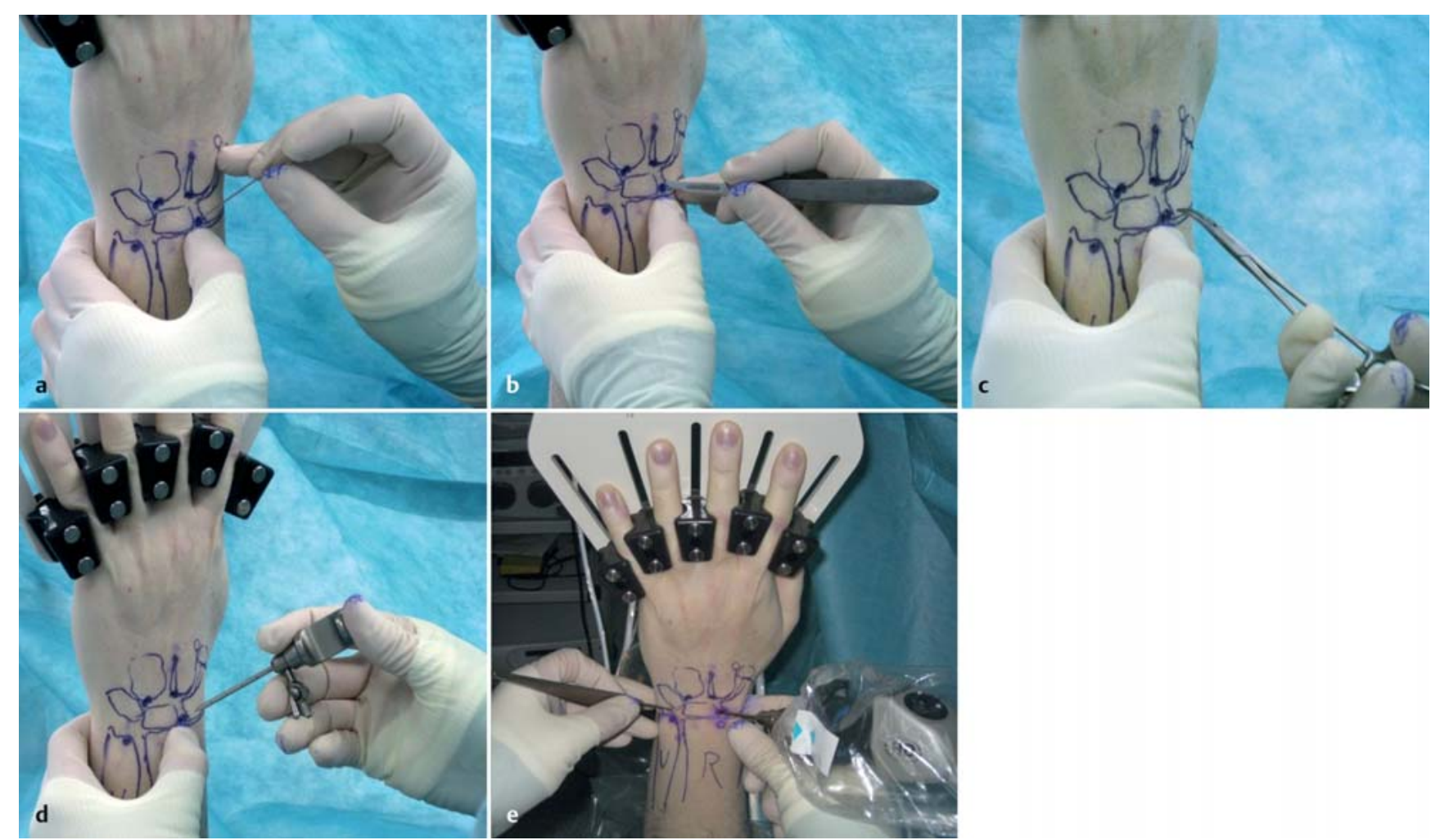

Abb. 6 a bis e Schaffen eines Portals zum Handgelenk. 


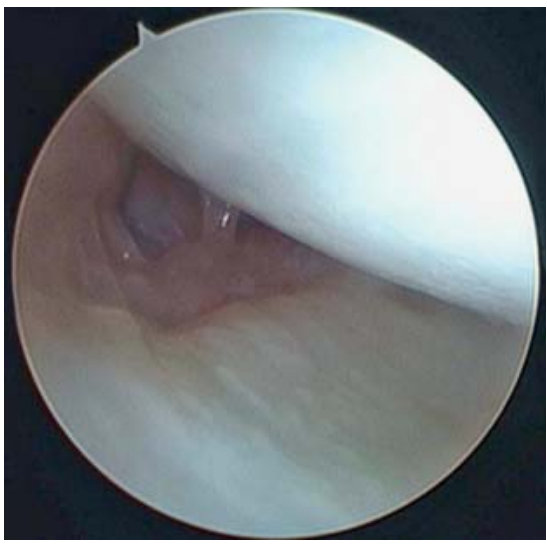

Abb. 7 Griffelfortsatz der Speiche und Gelenkspalt zwischen Speiche und Kahnbein.

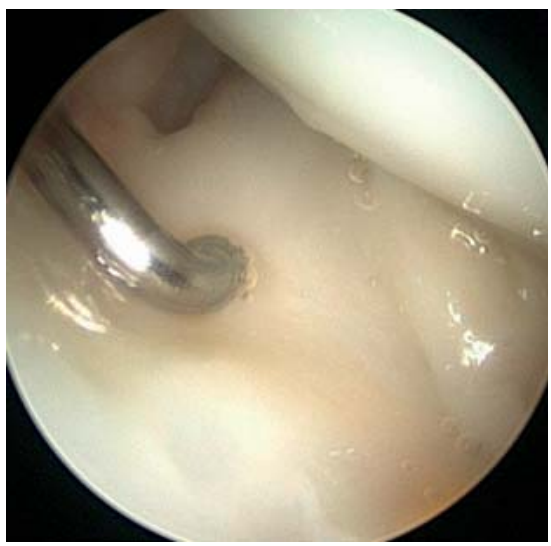

Abb.10 Testung des Trampolineffekts am TFCC.

portal wird üblicherweise in einem „soft spot" etwa $1 \mathrm{~cm}$ distal des Portals 3/4 platziert (MCR-Portal), ein Tasthaken kann auf gleicher Höhe ellenseitig hiervon eingebracht werden (MCU-Portal).

\section{Therapeutische arthroskopische Möglichkeiten}

Im Rahmen der diagnostischen Handgelenksarthroskopie besteht die Möglichkeit, gewisse pathologische Veränderungen ebenfalls arthroskopisch anzugehen, um somit eine Eröffnung des Handgelenks (Arthrotomie) zu vermeiden. Es handelt sich hierbei um folgende Situationen und Krankheitsbilder (ohne Anspruch auf Vollständigkeit), die unter gewissen Voraussetzungen arthroskopisch therapiert werden können:

\section{Glättung und Reparatur des TFCC}

Akute und degenerative Läsionen des TFCC werden, je nach ihrer Lokalisation und dem Ausmaß des Schadens, nach der sog. Palmer-Klassifikation eingeteilt [4]. Insbesondere zentrale Verletzungen

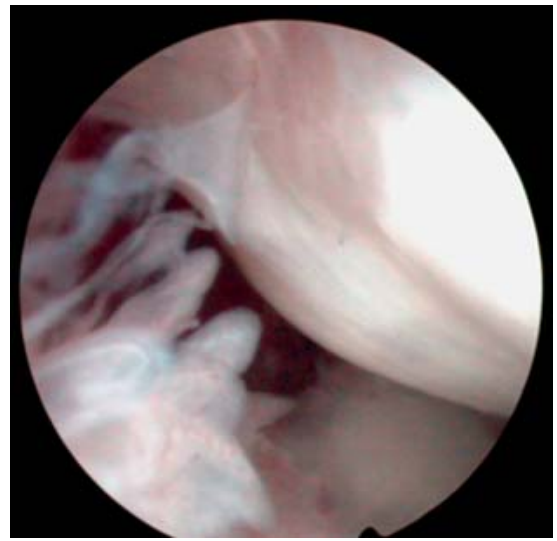

Abb. 8 Synovialitis/Synoviazotten.

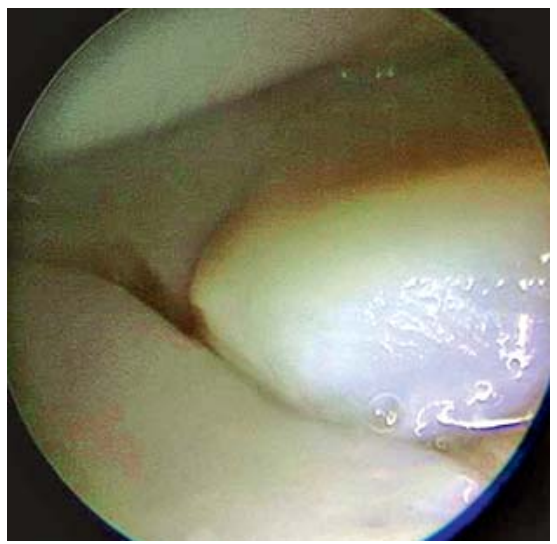

Abb. 11 Band zwischen Kahn- und Mondbein von mediokarpal.

werden débridiert und geglättet, da die fehlende Gefäßversorgung in diesem Bereich keine Heilung erwarten lässt (Abb. 12). Ein Abriss des TFCC an seiner ellenseitigen Befestigung (ellenseitiger Griffelfortsatz) kann jedoch häufig durch eine Ausziehnaht oder Verankerung im knöchernen Bereich der Elle behoben werden.

\section{Ulnokarpales Impaktationssyndrom}

Ist die Elle im Vergleich zur Speiche angeboren bzw. aufgrund eines Unfalls mit Frakturen oder Bandzerreißungen zu lang, liegt eine sog. Ulna-Plus-Varianz vor (Abb. 13). Stößt die Elle hierbei gegen die Handwurzel (genauer: Mondbein), so spricht man von einem ulnokarpalen Impaktationssyndrom. Dabei kommt der Discus triangularis im Laufe des Lebens immer wieder unter übermäßige Belastung und kann mit der Zeit aufgebraucht werden werden. Häufig liegen ein Knorpelschaden des darunterliegenden Ulnakopfs und des ellenseitigen Mondbeins vor. Durch arthroskopisches Abfräsen des durch den einhergehenden TFCC-De-

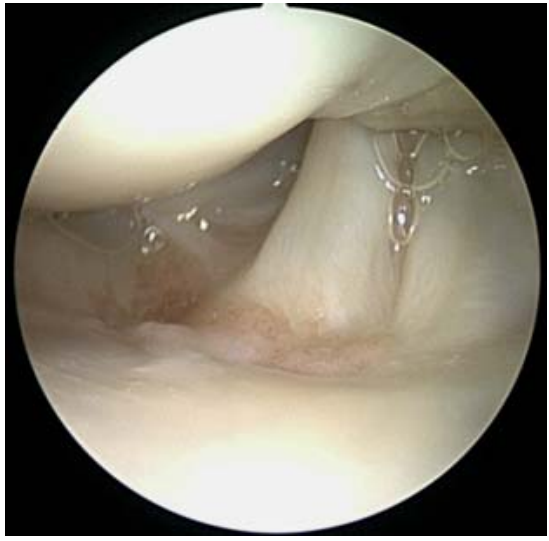

Abb.9 Palmare Handgelenksbänder.

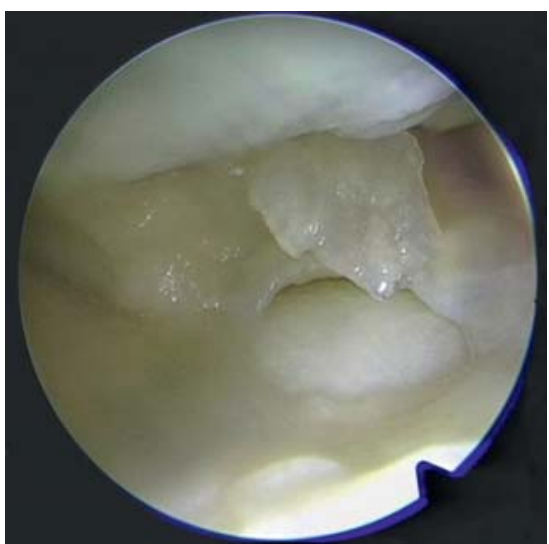

Abb. 12 Zentrales Loch im TFCC.

fekt freiliegenden Ellenkopfs kann die Elle um 3-4 mm verkürzt werden, was häufig zu einer deutlichen Beschwerdebesserung führt (Abb. 14) [2].

\section{Synovialektomie bei Synovialitis}

Aufgrund chronischer Entzündung wird die störende Gelenkschleimhaut operativ abgetragen, um Schmerzen, Schwellungszustände oder das Voranschreiten einer bestehenden Arthrose zu lindern. Normalerweise werden hierfür ShaverAufsätze mit einem Durchmesser zwischen 2,5 und $3,5 \mathrm{~mm}$ verwendet.

\section{Entfernung eines Handgelenksganglions}

Funktionell störende und schmerzhafte Handgelenksganglien können an bestimmten Lokalisationen arthroskopisch entfernt werden, indem mithilfe eines Shavers der Ganglionstiel im Bereich der Gelenkkapsel entfernt wird. Der subkutan gelegene Anteil des Ganglions schrumpft im Weiteren. Die Rezidivraten sind mit dem offen-chirurgischen Vorgehen gleichzusetzen [5]. 


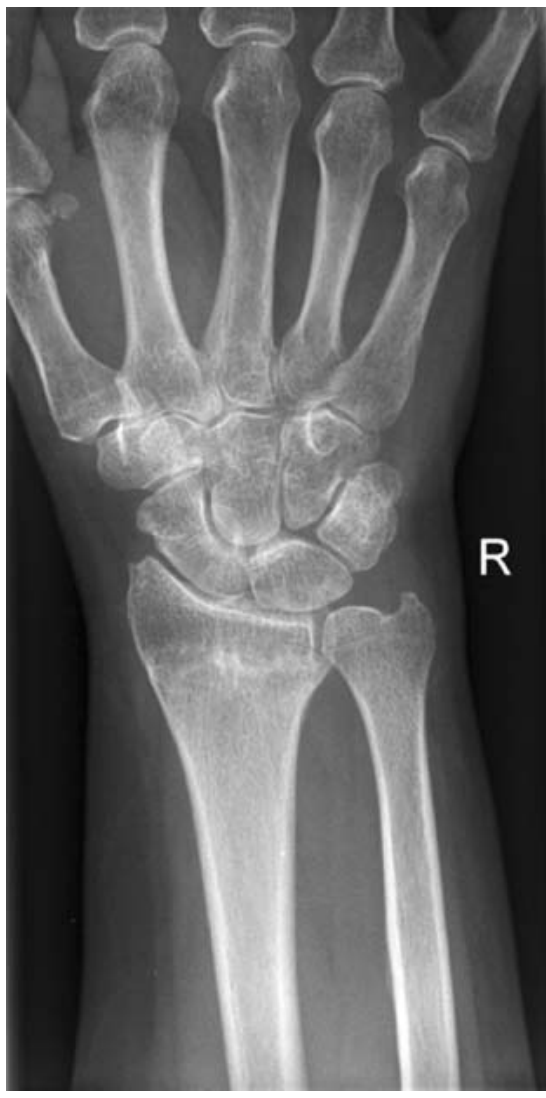

Abb. 13 Ulna-Plus-Varianz mit ulnokarpalem Impaktationssyndrom.

\section{Zystenausräumung}

Je nach Lokalisation und Bezug zum Gelenkspalt können Zysten arthroskopisch ausgeräumt und teilweise auch minimalinvasiv mit Knochenmaterial aufgefüllt werden (Abb. 15).

\section{Arthroskopisch assistierte Radiusfrakturversorgung}

Bei der Versorgung bestimmter distaler Radiusfrakturen mit Gelenkbeteiligung kann die anatomische stufenfreie Reposition und Osteosynthese der Fraktur durch direkte arthroskopische Betrachtung erleichtert werden [6]. Zudem wird die direkte Beurteilung intraartikulärer Bänder und des TFCC möglich. Die Distraktion des Handgelenks unterscheidet sich hierbei häufig von der oben beschriebenen Aufhängung, indem diese horizontal auf dem Handtisch erfolgt.

\section{Weitere arthroskopische}

Therapiemöglichkeiten

Neben den o.g. Verfahren können das Band zwischen Kahn- und Mondbein arthroskopisch genäht und einzelne Handwurzelknochen teilweise oder komplett

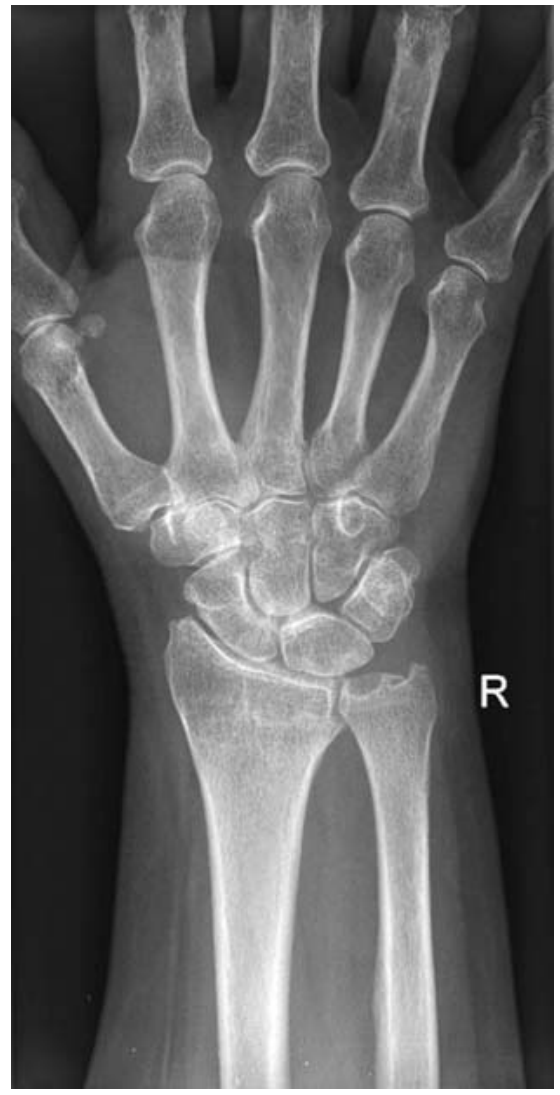

Abb. 14 Nach Abfräsen eines Teiles des Ulnakopfs über einen zentralen TFCC-Defekt.

entfernt werden (z.B. Hemitrapezektomie oder Trapezektomie bei Rhizarthrose), bis hin zum kompletten Wegfräsen der proximalen Handwurzelreihe bei Arthrose (Aufzählung hat keinen Anspruch auf Vollständigkeit). Des Weiteren verwenden wir die Handgelenksarthroskopie für Knorpelglättungen und die Entfernung freier Gelenkkörper.

\section{Schlussfolgerung}

Die Arthroskopie des Handgelenks hat sich seit Mitte der 1980er-Jahre kontinuierlich weiterentwickelt. Aus der anfänglich rein diagnostischen Zielsetzung entstanden zunehmend therapeutische Fragestellungen, welche teilweise nur durch technische Innovationen realisierbar wurden. Heutzutage bietet die Handgelenksarthroskopie eine weiter wachsende Palette an therapeutischen Möglichkeiten, welche teils unterstützend zu konventionell offenen Verfahren, teils eigenständig zu sehen sind.

\section{Literatur}

${ }^{1}$ Culp RW, Osterman L, Kaufmann RA. Wrist Arthroscopy: Operative Procedures. In: Green DP, Hotchkiss RN, Pedersen WC, Wolfe SW, eds. Green's Operative Hand Surgery. Phila-

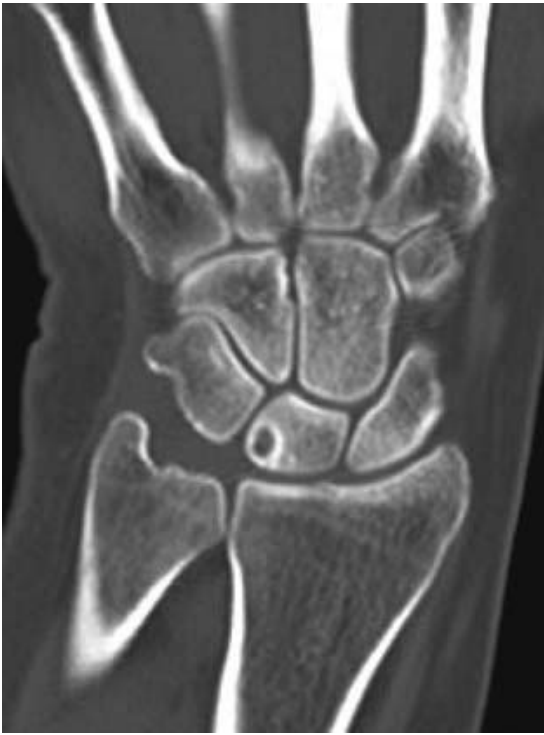

Abb. 15 Knochenzyste im Mondbein.

delphia: Elsevier Churchill Livingstone; 2005: 781-808

2 Elkowitz SJ, Posner MA. Wrist arthroscopy. Bull NYU Hosp Jt Dis 2006; 64: 156-165

${ }^{3}$ Ishii S, Palmer AK, Werner FW et al. An anatomic study of the ligamentous structure of the triangular fibrocartilage complex. J Hand Surg Am 1998; 23: 977-985

${ }^{4}$ Geissler W. Preface. In: Geissler W, ed. Wrist Arthroscopy. New York: Springer; 2005: vii

${ }^{5}$ Ishii S, Palmer AK, Werner FW et al. An anatomic study of the ligamentous structure of the triangular fibrocartilage complex. J Hand Surg Am 1998; 23: 977-985

${ }^{6}$ Kang L, Akelman E, Weiss AP. Arthroscopic versus open dorsal ganglion excision: a prospective, randomized comparison of rates of recurrence and of residual pain. J Hand Surg Am 2009; 33: 471-475

${ }^{7}$ Khanchandani P, Badia A. Functional outcome of arthroscopic assisted fixation of distal radius fractures. Indian J Orthop 2013; 47: 288-294

${ }^{8}$ VBM Medizintechnik GmbH Blutsperre Leitfaden 2009. Im Internet: URL: http://www. $\mathrm{vbm}$-medical.de/cms/files/blutsperre_leitfaden_2.0_05.09_de.pdf; Stand 07.02.2015

\section{Dr. med. Oliver Lotter, MBA}

Leitender Oberarzt

\section{Prof. Dr. med. Hans-Eberhard} Schaller

Direktor der Klinik

Klinik für Plastische, Hand-, Rekonstruktive und Verbrennungschirurgie an der Eberhard-Karls-Universität Berufsgenossenschaftliche Unfallklinik Tübingen

Schnarrenbergstraße 95 72076 Tübingen

OLotter@bgu-tuebingen.de 